\title{
Fuel, electricity, ER and HER2-a hybrid-car model of breast cancer
}

\section{Shigehira Saji, Fumiaki Sato and Naoto T. Ueno}

Recent progress in our understanding of breast cancer biology resulting from studies using different molecular profiling techniques has yielded a complex picture of the mechanisms driving tumour progression. At the same time, a variety of different molecular-targeting agents have been developed for the treatment of patients with breast cancer. It has gradually become difficult for many of us to understand which pathways are inhibited by which targeted drugs. This progress was discussed in a recent article by Rimawi and Osborne (Breast cancer: Blocking both driver and escape pathways improves outcomes. Nat. Rev. Clin. Oncol. 9, 133-134). ${ }^{1}$

We propose here that the complex breast cancer model can be understood by comparing it to a hybrid vehicle with two power sources, such as the Toyota Prius. The Prius uses an electric motor and a gasoline-powered engine simultaneously or independently, and each power source affects the other. Also, the car has a monitor showing which power source is currently active and how the two power sources work together when the car is running. According to our current knowledge about breast cancer biology, the two main power sources in breast cancer are the oestrogen receptor
(ER) and HER2. Figure 1 shows how these two 'power sources' act in concert with one other to promote breast cancer tumorigenesis. In the absence of oestradiol (petrol in our analogy), the membrane receptoractivated PI3K pathway and the MAPK pathway (motor and transmission) cause phosphorylation of ER (engine) and activate ER downstream genes (transmission). ${ }^{2,3}$ The impact on breast cancer of endocrine therapy, cytotoxic therapies, and targeted therapies, including antiangiogenesis agents, are illustrated in Figure 1b. This hybrid car analogy can also be used to illustrate the basic mechanisms of action in the recently reported BOLERO- 2 trial, ${ }^{4}$ in which patients with ER-positive breast cancer were treated with a combination of the mTOR inhibitor everolimus and the aromatase inhibitor exemestane (Figure 1c). Of course, if we had a monitor analogous to the Prius's monitor indicating the power sources active in each patient's breast tumour, treatment choice would become dramatically easier.

Our simple hybrid car model could be a useful educational model for clinical oncologists who need to understand breast cancer biology and how best to employ the newly developed targeted therapies.
Department of Target Therapy Oncology, Kyoto University Graduate School of Medicine, 54 Shogoin Kawahara-cho, Sakyo-ku, Kyoto 606-8507, Japan (S. Saji, F. Sato). Morgan Welch Inflammatory Breast Cancer Research Program and Clinic, Section of Translational Breast Cancer Research, Department of Breast Medical Oncology, The University of Texas MD Anderson Cancer Center, 1515 Holcombe Boulevard, Unit 1354, Houston, TX 77030, USA (N. T. Ueno).

Correspondence to: S. Saji

ss-saji@wa2.so-net.ne.jp

Competing interests

S. Saji declares associations with the following companies: Chugai/Roche, Novartis. F. Sato declares associations with the following companies: AstraZeneca, Chugai/Roche. N. T. Ueno declares an association with the following company: Kyowa Hakko Kirin. See the article online for full details of the relationships.

1. Rimawi, M. F. \& Osborne, C. K. Breast cancer: Blocking both driver and escape pathways improves outcomes. Nat. Rev. Clin. Oncol. 9, 133-134 (2012).

2. Miller, T. W., Balko, J. M. \& Arteaga, C. L. Phosphatidylinositol 3-kinase and antiestrogen resistance in breast cancer. J. Clin. Oncol. 29, 4452-4461 (2011).

3. Osborne, C. K. \& Schiff, R. Mechanisms of endocrine resistance in breast cancer. Ann. Rev. Med. 62, 233-247 (2011).

4. Baselga, J. et al. Everolimus in postmenopausal hormone-receptor-positive advanced breast cancer. N. Engl. J. Med. 366, 520-529 (2011). 


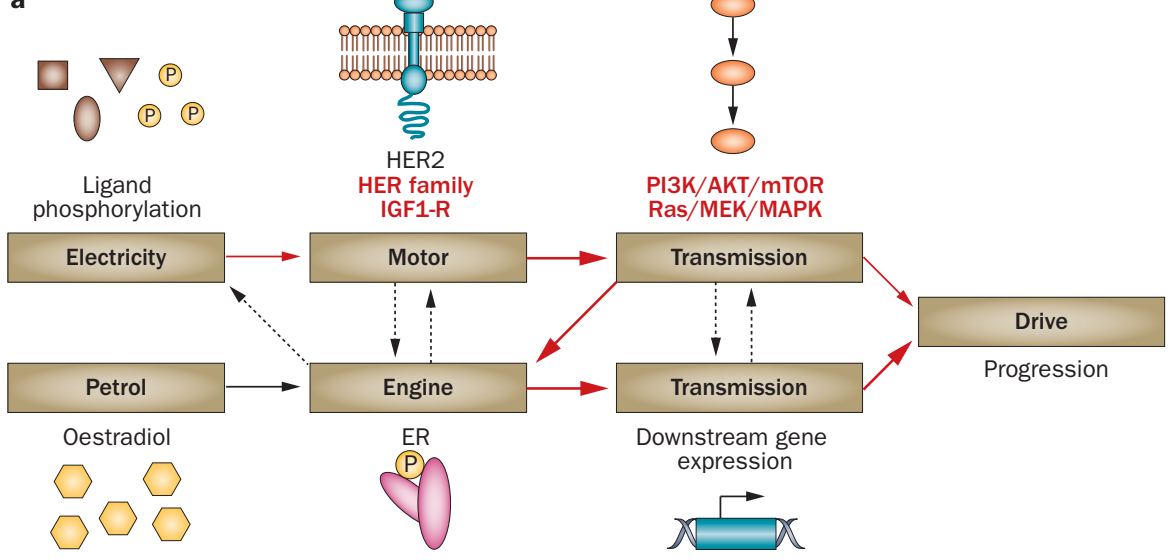

4 Figure 1 | Similarity between breast cancer and hybrid car. a | In the absence of oestradiol (petrol), PI3K and MAPK pathways (motor and transmission) cause phosphorylation of ER (engine) and activate ER downstream gene expression (transmission). b | Sites of action of various drugs. c| Basic mechanism of action of combination of everolimus (mTOR inhibitor) and exemestane (aromatase inhibitor) in ER-positive breast cancer. Colour of arrows indicates dependency on each 'power source'. Abbreviations: ER, oestrogen receptor; SERD, selective oestrogen receptor downregulator; SERM, selective oestrogen receptor modulator.

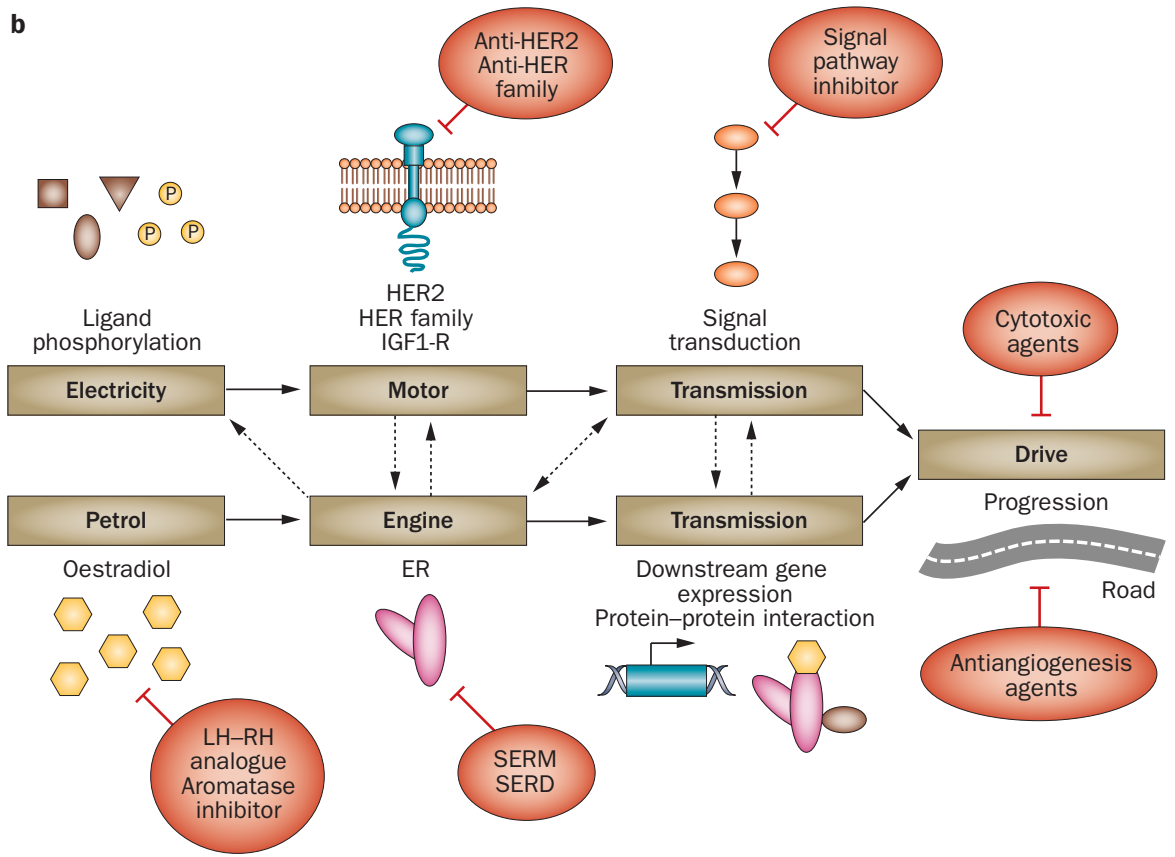

c

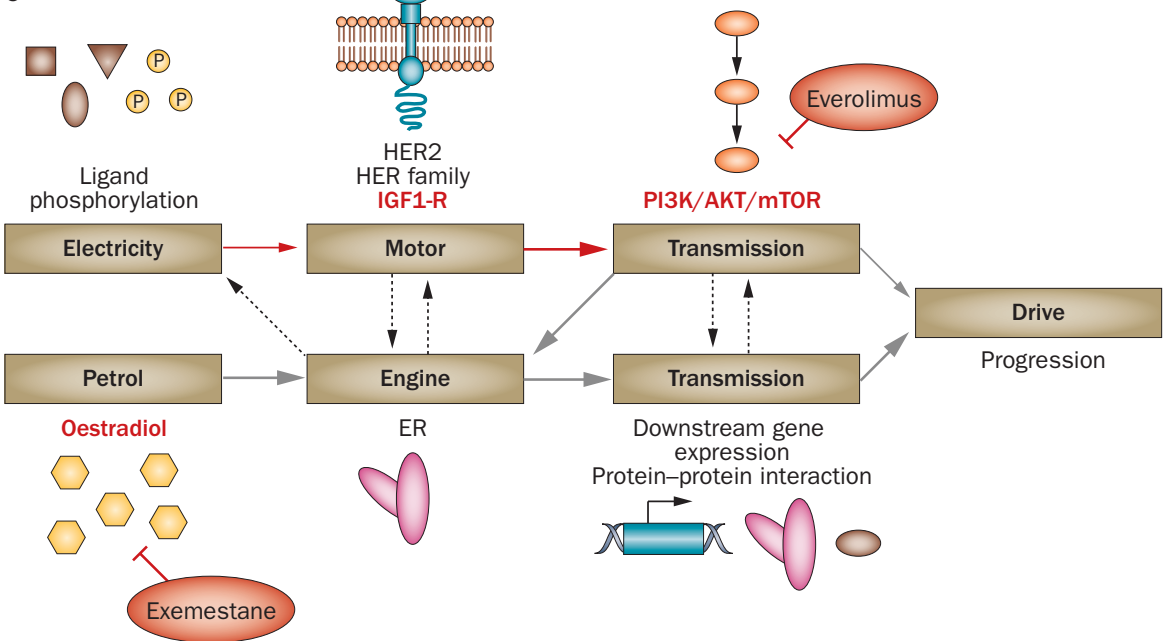

